\title{
LES LARVES HÉPATIQUES DE CESTODE parasites du grand campagnol Arvicola terrestris en Auvergne (France).
}

\author{
S. DEBLOCK* et A. F. PÉTAVY**
}

RÉSUMÉ. L'autopsie de 943 Arvicola terrestris scherman Shaw (Mammifère microtidé) adultes capturés en Auvergne (départements du Puy-de-Dôme et du Cantal) au cours des années 1981 et 1982, met en évidence l'existence de larves de quatre espèces différentes de tænia parasites du foie, avec les fréquences suivantes : 37 fois Cysticercus fasciolaris $(3,92 \%), 23$ fois Echinococcus multilocularis $(2,44 \%), 11$ fois Cysticercus tenuicollis $(1,16 \%)$ et 10 fois C. laticollis $(1,06 \%)$. C. longicollis est présent une fois $(0,10 \%)$ comme parasite de la cavité abdominale. Il n'existe habituellement qu'une larve par foie mais le parasitisme multiple n'est pas exclu [2 larves de $C$. fasciolaris (2 cas), 3 larves (1 cas) ou 5 ( 1 cas). 5 larves de $C$. tenuicollis $(1$ cas)]. Deux parasites d'espèces différentes peuvent s'associer $(C$. fasciolaris $+C$. laticollis $: 1$ cas. $C$. fasciolaris $+E$. multilocularis $: 3$ cas $)$; de ce fait, un total de 77 foies de rongeurs sont parasités sur les 943 examinés, soit 8,16\%. Le rôle des grands campagnols dans l'épidémiologie des cinq espèces de cestodes, toutes de Mammifères carnivores, est discuté en fonction des observations faites dans le temps au niveau des six différents lieux de capture des hôtes. Larves et lésions hépatiques font l'objet de descriptions anatomiques et histologiques comparées dans un but diagnostique. Ces investigations sont rendues nécessaires par le fait que 14 des 23 larves alvéolaires sont des larves immatures très peu développées, mono- ou pauci-vésiculaires, d'un diamètre approximatif de 300 à $1500 \mu \mathrm{m}$ et non fertiles : leur diagnostic spécifique, compte tenu de ces aspects inhabituels, pourrait a priori prêter à discussion. La réaction de l'adventice n'est révélatrice de la nature d'aucune des quatre espèces de cestode hépatiques rencontrées.

\section{The hepatic larvae of Cestode parasiting the vole rat Arvicola terrestris in Auvergne (France).}

SUMMARY. The autopsy of 943 vole rats Arvicola terrestris scherman Shaw (Mammal Microtidae) from Auvergne (Departments of Puy-de-Dôme and Cantal) obtained for the year 1981 and 1982 shows evidence of four different species of hepatic cestode larvae. Cysticercus fasciolaris occured in $37(3.92 \%)$, Echinococcus multilocularis in $23(2.44 \%)$, C. tenuicollis in $11(1.16 \%)$ and C. laticollis in $10(1.06 \%)$. C. longicollis occured in one $(0.10 \%)$ as parasite of the abdominal cavity. There is usually just one larva for a liver but a multiple parasitism is not excluded : 2 larvae of $C$. fasciolaris ( 2 cases), or 3 or 5 larvae (one case each); 5 larvae of $C$.tenuicollis ( 1 case). Mixed parasitism also is possible $(C$. fasciolaris + C. laticollis $: 1$ case. C. fasciolaris + E. multilocularis :

\footnotetext{
* Laboratoire de Parasitologie. U.E.R. de Pharmacie de Lille, rue du Professeur-Laguesse, F 59045 Lille Cedex.

** Laboratoire de Parasitologie, Facultés de Médecine et de Pharmacie, 8 avenue Rockefeller, F 69373 Lyon.
}

Accepté le 28 janvier 1983 . 
3 cases). So 77 livers $(8.16 \%)$ are parasited. The role of the vole rat in the epidemiology of the five species of cestodes is discussed. Anatomical and histological aspects are described for each species to make certain the differential and specific diagnosis of the young and frequent E. multilocularis larvae.

\section{Introduction}

Cinq cas humains autochtones d'échinococcose alvéolaire hépatique ont révélé l'existence d'un nouveau foyer de la parasitose en France dans le Massif Central à partir de 1970. L'étude épidémiologique du foyer auvergnat a commencé par la démonstration de l'existence de l'hôte définitif (Pétavy et coll., 1980) ; elle se poursuit par la démonstration de l'existence d'un hôte intermédiaire. A cet effet, la capture de 943 Arvicola terrestris scherman Shaw (Mammifère Microtidé ; grand campagnol ou rat taupier) est réalisé en 1981 et 1982 en divers points de la province d'Auvergne, à proximité des lieux d'origine soit des cas humains, soit des renards porteurs du tænia échinocoque alvéolaire. L'examen des foies des rongeurs à l'autopsie montre, outre l'existence effective d'hydatides alvéolaires, celle de larves d'autres espèces de Tænia. Nature, répartition géographique, fréquence de ces parasites font l'objet de notre étude. Les aspects macro- et micro-scopiques comparés des cystiques et de leurs enveloppes sont pris spécialement en considération, de façon à justifier le diagnostic différentiel des larves alvéolaires jeunes, assez fréquentes dans notre série de parasites. Celles-ci offrent en effet un aspect immature dont l'identification spécifique ne devient possible qu'après comparaison avec les structures d'autres cystiques.

\section{Matériel et méthodes}

Lieux et modes de piégeage des campagnols sont précisés dans un article différent (Pétavy et coll., 1983), de même que les modalités de l'examen des foies et des lésions hépatiques. Autopsies et examens parasitologiques sont menés sur le terrain selon les techniques usuelles de routine en la matière. Toute lésion macroscopique suspecte au niveau des foies est fixée sans délai dans un réactif formolé en vue d'un examen détaillé ultérieur au laboratoire, et d'un examen histologique éventuel, chaque fois que l'exactitude du diagnostic l'exige.

Les mensurations rapportées dans les divers paragraphes ne portent que sur la partie du matériel parasitaire récolté considérée comme la plus favorable ou la mieux représentative, et non sur la totalité des préparations examinées en vue des déterminations spécifiques ; toutefois ces mensurations sont toujours sufisamment multipliées pour acquérir une valeur significative.

\section{Résultats des autopsies}

Le foie de 77 rongeurs sur 943 campagnols autopsiés, soit 8,16\%, héberge une ou plusieurs espèces de larves hépatiques de cestode appartenant à quatre espèces différentes. Le synopsis des larves déterminées s'établit comme suit : 
A - Larves hépatiques

1. Cysticercus fasciolaris, larve du Taenia taeniaeformis (Batsch, 1786), parasite à l'état adulte des Félidés, Canidés et, éventuellement, Mustélidés.

2. Cysticercus hypudaei Leuckart, 1857, larve du Taenia tenuicollis Rud., 1819 parasite à l'état adulte de Mustélidés.

3. Cysticercus laticollis (Batsch, 1786), larve du Taenia laticollis Rud., 1819 parasite à l'état adulte du lynx et de la genette.

4. Larve échinococcique alvéolaire de l'Echinococcus multilocularis Leuckart, 1863 parasite à l'état adulte du renard, du chien et du chat.

B - Larves de la cavité péritonéale.

Cysticercus longicollis Rud., 1819, larve du Taenia crassiceps (Zeder, 1800) parasite à l'état adulte du renard.

\section{1 - Cysticercus fasciolaris}

Le cystique est observé 37 fois lors des 943 autopsies, soit chez 3,92 \% des hôtes recueillis ( 15 mâles et 22 femelles adultes). Il n'existe qu'une larve par foie en général, rarement deux ( 2 cas), trois ( 1 cas) ou cinq (1 cas). Dans un cas le parasite est associé à un Cysticercus laticollis, et dans trois cas à des lésions d'hydatidose alvéolaire (Pl. I, fig. 1).

Tableau I. - Prévalence de $C$. fasciolaris

en fonction des diverses communes de piégeage des Arvicola.

\begin{tabular}{|c|c|c|c|c|c|c|c|}
\hline \multirow{2}{*}{$\begin{array}{l}\text { Dépar- } \\
\text { tements }\end{array}$} & \multirow{2}{*}{ Communes } & \multicolumn{2}{|c|}{ Dates } & Nombre & Total & \multirow{2}{*}{\multicolumn{2}{|c|}{$\begin{array}{l}\text { Foies parasités } \\
\text { Nombre } \%\end{array}$}} \\
\hline & & \multicolumn{4}{|c|}{ des captures } & & \\
\hline \multirow{5}{*}{$\begin{array}{l}\text { Puy- } \\
\text { de- } \\
\text { Dôme }\end{array}$} & Voingt & Août & 1981 & & 50 & 2 & 4 \\
\hline & Giat & Mars & 1982 & & 3 & $\overline{0}$ & - \\
\hline & Zanières & $\begin{array}{l}\text { Août } \\
\text { Mars }\end{array}$ & $\begin{array}{l}1981 \\
1982\end{array}$ & $\begin{array}{r}36 \\
161\end{array}$ & 197 & $\begin{array}{l}2 \\
6\end{array} 8$ & 4,2 \\
\hline & Mareuge & $\begin{array}{l}\text { Avril } \\
\text { Juill. }\end{array}$ & $\begin{array}{l}1982 \\
1982\end{array}$ & $\begin{array}{l}93 \\
94\end{array}$ & 187 & $\begin{array}{l}0 \\
2\end{array}$ & 1,06 \\
\hline & $\begin{array}{l}\text { Égliseneuve } \\
\text { d'Entraigues }\end{array}$ & $\begin{array}{l}\text { Juill. } \\
\text { Août }\end{array}$ & $\begin{array}{l}1982 \\
1982\end{array}$ & $\begin{array}{l}33 \\
57\end{array}$ & 90 & $\begin{array}{l}1 \\
0\end{array}$ & 1,1 \\
\hline \multirow[t]{3}{*}{ Cantal } & Allanche & Août & 1981 & 37 & & 2) & \\
\hline & & $\begin{array}{l}\text { Oct. } \\
\text { Janv. } \\
\text { Mai }\end{array}$ & $\begin{array}{l}1981 \\
1982 \\
1982\end{array}$ & $\begin{array}{r}167 \\
74 \\
87\end{array}$ & 365 & \begin{tabular}{l|l}
8 & 19 \\
1 & 19 \\
8 &
\end{tabular} & 5,2 \\
\hline & Condat & $\begin{array}{l}\text { Mars } \\
\text { Mai }\end{array}$ & $\begin{array}{l}1982 \\
1982\end{array}$ & $\begin{array}{l}27 \\
24\end{array}$ & 51 & $\left.\begin{array}{l}3 \\
2\end{array}\right\}$ & 9,8 \\
\hline
\end{tabular}

Le tableau $I$ montre que l'endémie à $C$. fasciolaris est assez uniformément répartie dans les divers biotopes considérés ; le parasite paraît donc bien implanté en Auvergne. 
TABleau II. - Prévalence de $C$. fasciolaris en fonction du mois de capture des hôtes (tous lieux de capture confondus).

\begin{tabular}{lccc}
\hline $\begin{array}{c}\text { Dates des } \\
\text { captures }\end{array}$ & $\begin{array}{c}\text { Nombre des } \\
\text { captures }\end{array}$ & $\begin{array}{c}\text { Nombre de } \\
\text { C. fasciolaris }\end{array}$ & Pourcentages \\
\hline Août 1981 & 123 & 6 & 4,87 \\
Oct. 1981 & 167 & 8 & 4,79 \\
Janv. 1982 & 74 & 1 & 1,35 \\
Mars 1982 & 191 & 9 & 4,71 \\
Avril 1982 & 93 & 0 & 9 \\
Mai 1982 & 111 & 10 & 2,36 \\
Juil. 1982 & 127 & 3 & 0 \\
Août 1982 & 57 & 0 & \\
\hline
\end{tabular}

Le tableau II montre que l'incidence du parasite varie peu dans le temps et qu'elle se maintient stable dans les biotopes favorables.

L'aspect macroscopique du parasite est uniforme : kyste hépatique bien circonscrit, ovoïde ou sphérique, ferme, de couleur blanc-jaunâtre lorsqu'il saille de part et d'autre du lobe atteint. Ses tailles extrêmes sont de $6 \times 7 \times 7$ à $15 \times 15 \times 10 \mathrm{~mm}$ de diamètre. A l'ouverture du kyste, la strobilisation de la larve est plus ou moins achevée selon les exemplaires, et sa longueur varie de 15 à $130 \mathrm{~mm}$. La larve envahit la totalité de l'espace kystique interne. Les exemplaires immatures se présentent dans notre série de parasites comme des cystiques liquidiens creux dont la vésicule extériorise le strobile entre elle-même et l'adventice. Aucun cystique embryonnaire encore dépourvu de scolex n'a été repéré dans les foies examinés.

Dimensions. - Scolex : 1200 à $1500 \mu \mathrm{m}$ de $\varnothing$ sur $1500 \mu \mathrm{m}$ de long : ventouses : 375 à $400 \mu \mathrm{m}$ de $\varnothing$; rostre invaginé ou non selon les exemplaires avec 2 couronnes de 15 à 18 crochets (ex. : 2 fois 15,1 fois 16, 2 fois 17 et 3 fois 18) (tableau III).

Tableau III. - Taille des crochets (en $\mu \mathrm{m}$ ) de $C$. fasciolaris des Arvicola en Auvergne.

\begin{tabular}{cccc}
\hline$\mu \mathrm{m}$ & longueur & hauteur & largeur de la garde \\
\hline Grands crochets & $430-469$ & $165-195$ & $65-85$ \\
Petits crochets & $250-287$ & $105-120$ & $53-88$ \\
\hline
\end{tabular}

Aspect histologigue du cystique. - La vésicule larvaire est bordée d'une membrane tégumentaire périphérique continue épaisse de 1,5 à $4 \mu \mathrm{m}$ environ et anhiste. Elle est tapissée intérieurement d'un parenchyme nucléé de 50 à $90 \mu \mathrm{m}$ d'épaisseur, plus dense au contact du tégument et plus lâche au contact de la lumière du kyste. Les deux zones ainsi différenciées sont séparées au voisinage de l'implantation du strobile sur la vésicule par une mince assise de fibrilles musculaires bien individualisées et communes aux deux formations anatomiques.

Le parenchyme contient en outre quelques corpuscules calcaires et un réseau 
de canaux excréteurs au voisinage desquels se situent des solénocytes. Scolex et strobile bourgeonnent à l'extérieur de la vésicule larvaire et se situent directement en regard de l'adventice de l'hôte; la membrane tégumentaire primitive fournit la vésicule caudale. Le tégument du cystique non en contact avec l'adventice acquiert un revêtement continu et dense de microtriches longs de $2,5 \mu \mathrm{m}$ au moins. Le kyste réactionnel de l'hôte est mince ou très mince par rapport au grand diamètre de la larve ; son épaisseur varie selon le diamètre et selon la larve considérés. (exemple : 65 à $110 \mu \mathrm{m} ; 90$ à $260 \mu \mathrm{m} ; 150$ à $210 \mu \mathrm{m})$. La larve enkystée paraît bien tolérée par l'hôte ; le strobile est parfois enveloppé en tout ou partie d'un magma caséeux blanchâtre qui n'altère pas l'intégrité tissulaire du parasite; il n'a pas été observé de processus d'involution.

C. fasciolaris n'infeste pas les renards en Auvergne (Pétavy et Deblock, 1980) malgré sa fréquence élevée dans les foies des campagnols.

\section{2 - Cysticercus tenuicollis}

Le cystique est observé 12 fois lors des 943 autopsies, soit chez 1,27 \% des hôtes capturés (5 mâles et 7 femelles adultes). Il n'existe qu'une larve par foie en général, parfois deux. Un foie en contenait cinq. Il n'a pas été observé d'association du cystique avec d'autres espèces dans un même foie ( $P l$. I, fig. 2).

Tableau IV. - Prévalence de $C$. tenuicollis dans les divers lieux de capture des hôtes.

\begin{tabular}{|c|c|c|c|c|}
\hline \multirow{2}{*}{$\begin{array}{l}\text { Dépar- } \\
\text { tement }\end{array}$} & \multirow{2}{*}{ Communes } & \multirow{2}{*}{$\begin{array}{l}\text { Nombre des } \\
\text { captures }\end{array}$} & \multicolumn{2}{|c|}{ Foies parasités } \\
\hline & & & Nombre & $\%$ \\
\hline \multirow{5}{*}{$\begin{array}{c}\text { Puy- } \\
\text { de- } \\
\text { Dôme }\end{array}$} & Voingt & 50 & 0 & - \\
\hline & Giat & 3 & 0 & - \\
\hline & Zanières & 197 & 3 & 1,52 \\
\hline & Mareuge & 187 & 7 & 3,74 \\
\hline & Égliseneuve d'Entraigues & 90 & 0 & - \\
\hline \multirow[t]{2}{*}{ Cantal } & Allanche & 365 & 2 & 0,54 \\
\hline & Condat & 51 & 0 & - \\
\hline
\end{tabular}

Le tableau $I V$ montre que l'endémie à $C$. tenuicollis est irrégulièrement distribuée en Auvergne ; certains biotopes paraissent plus favorable à la parasitose, vraisemblablement du fait de la présence locale d'une population de Mustélidés réceptifs disséminant les onchosphères du parasite.

Le tableau $V$ paraît montrer que les foyers favorables sont capables de se maintenir en activité tout au long de l'année.

L'aspect macroscopique du foie parasité est uniforme : kyste hépatique bien circonscrit, régulièrement sphérique sauf exception, ferme, de petite taille $(1,8 \mathrm{~mm}$ 
TABleau V. - Prévalence de C. tenuicollis

en fonction du mois de capture des hôtes (tous lieux de capture confondus).

\begin{tabular}{|c|c|c|c|}
\hline Dates & $\begin{array}{l}\text { Nombre de } \\
\text { captures }\end{array}$ & $\begin{array}{l}\text { Nombre de } \\
\text { C. tenuicollis }\end{array}$ & $\%$ \\
\hline Août 1981 & 123 & 1 & 0,81 \\
\hline Oct. & 167 & 0 & - \\
\hline Janv. 1982 & 74 & 1 & 1,35 \\
\hline Mars & 191 & 3 & 1,57 \\
\hline Avril & 93 & 6 & 6,45 \\
\hline $\mathrm{Mai}$ & 111 & 0 & - \\
\hline \multirow{3}{*}{ Joûl. } & 127 & 1 & 0,78 \\
\hline & 57 & 0 & - \\
\hline & 943 & 12 & 1,27 \\
\hline
\end{tabular}

de $\varnothing)$ et situé en profondeur dans le parenchyme ; de ce fait les altérations de surface subies par l'organe sont nulles ou des plus discrètes ; il est donc nécessaire de couper le foie en tranches minces pour mettre le parasite en évidence.

Le cystique apparaît comme une vésicule creuse et liquidienne comportant un scolex monté sur un court pédoncule né de la membrane vésiculaire à partir d'une invagination en doigt de gant ; ventouses et rostre invaginé se font face en périphérie $\mathrm{du}$ pertuis de l'invagination. Le parenchyme est toujours très riche en corpuscules calcaires ovalaires. Aucun cystique embryonnaire encore dépourvu de scolex n'a été repéré dans les foies autopsiés.

Dimensions. - Le scolex invaginé mesure 500-800 $\mu \mathrm{m}$ de long $\times 300-400 \mu \mathrm{m}$ de diamètre. Ventouses rostre et crochets ne deviennent visibles que par écrasement du scolex en gomme-chloral, ou sur coupes histologiques. Ventouses : 110-115 $\mu \mathrm{m}$ de $\varnothing$. Coussin du rostre : $60 \mu \mathrm{m}$ de $\varnothing$. Deux couronnes jointives de 23 crochets chacunes, subégaux, mesurant 19 à $23 \mu \mathrm{m}$ de long $\times 11$ à $13 \mu \mathrm{m}$ de haut ; la garde spatulée mesure $6,5 \mu \mathrm{m}$ d'épaisseur et $5 \mu \mathrm{m}$ de largeur. La silhouette des crochets des deux rangs est légèrement dissemblable. Dollfus, 1961 a fourni les variations de nombre et de taille des crochets larvaires récoltés en divers points du territoire national.

Aspect histologique DU CySTiQUe. - La vésicule parasitaire plus ou moins décollée de l'adventice est bordée d'une membrane tégumentaire périphérique continue ; au niveau de l'hémisphère opposé au scolex, cette membrane est fine $(1,5 \mu \mathrm{m})$, lisse ou onduleuse, anhiste et colorable. Sa face externe, lorsqu'elle n'est pas au contact de l'adventice, est tapissée d'un revêtement dense de microtriches ténus longs de $6,5 \mu \mathrm{m}$; la face interne est en contact avec une couche parenchymateuse dense devenant plus lâche au fur et à mesure qu'elle s'élève, et épaisse de 15 à $20 \mu \mathrm{m}$. Elle est riche en noyaux sous-tégumentaires ainsi qu'en lacunes de $13 \times 20$ ou de $15 \mu \mathrm{m}$ de $\varnothing$ ayant hébergé des corpuscules calcaires ovalaires. Au niveau du second hémisphère, le tégument subit plusieurs modifications, de plus en plus accusées au fur et à mesure qu'on se rapproche du pertuis d'invagination du scolex : 1) un épaississement progressif, jusqu'à 7 à $8 \mu \mathrm{m}$, de la cuticule; 2) une disparition de la couche ciliée externe. 
Sous le tégument s'individualisent : a) une couche fibrillaire formée d'une assise unique et linéaire de fibrilles fines $(0,3 \mu \mathrm{m}$ environ) ; b) une couche musculaire faite d'une assise unique et linéaire de fibres de $4 \times 2 \mu \mathrm{m}$ de $\varnothing ; c)$ des canaux néphridiens et des solénocytes. Au-delà se situe un parenchyme lâche.

Une fine membrane d'aspect pellucide de $1 \mu \mathrm{m}$ d'épaisseur tapisse certaines surfaces de l'adventice à proximité du parasite ; nous la considérons de nature conjonctive. Ailleurs cette adventice est à nu ou en contact avec un exsudat fibrineux léger ou dense présentant parfois des vacuoles de résorption au contact du parasite ou de l'hôte.

\section{3 - Cysticercus laticollis}

Le cystique de cette espèce est observé dans 10 foies sur 943 autopsies, soit $1,06 \%$ des hôtes capturés ( 5 mâles et 5 femelles adultes). Il existe une larve par organe sauf une fois où deux larves coexistent. L'espèce s'associe avec $C$. fasciolaris dans un cas. $P l . I$, (fig. 3).

Le parasite n'a été mis en évidence que dans trois communes (tableau VI).

TABleau VI. - Prévalence de $C$. laticollis

dans les lieux de capture des Arvicola. Incidence des mois de l'année.

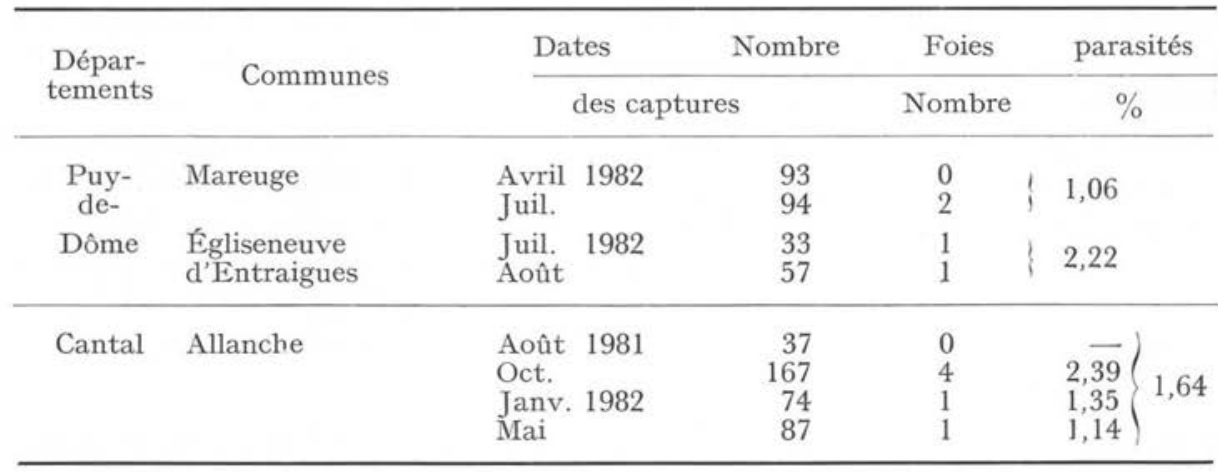

L'endémie paraît donc assez localisée géographiquement en Auvergne, l'hôte définitif étant rare (genette) ; mais dans les biotopes favorables comme à Allanche, elle s'y manifeste à un taux de prévalence assez élevé, stable dans le temps.

L'aspect macroscopique du parasite est uniforme : kyste hépatique volumineux régulièrement sphérique ou sub-sphérique, de couleur blanc-jaunâtre lorsqu'il fait partiellement saillie hors du lobe hépatique. Il mesure $6 \times 7 \mathrm{~mm}$ ou $6 \times 7 \times 8 \mathrm{~mm}$ de $\varnothing$. Son aspect général peut donc évoquer $C$. fasciolaris. Toutefois l'ouverture du kyste fait découvrir une vaste cavité liquidienne dont la paroi donne naissance à un gros scolex dressé, pédonculé sur un cou long de 1,5 à $2 \mathrm{~mm}$ et dont les ventouses sont bien apparentes ; en fait, ce scolex est issu d'une invagination en doigt de gant de la membrane vésiculaire comme dans le cas de la précédente espèce. 
Dimensions. - Scolex : 600-800 $\mu \mathrm{m}$ de long $\times 600-700 \mu \mathrm{m}$ de $\varnothing$. Ventouses relativement petites, circulaires, de 130-190 $\mu \mathrm{m}$ (moyenne : $160 \mu \mathrm{m}$ ). Bouquet des crochets du rostre invaginé : $370 \times 320-370 \mu \mathrm{m}$ de $\varnothing$, situé en avant de la ligne des ventouses ; sa présence entraîne une conformation en dôme assez caractéristique de la partie antérieure du scolex. Cou long de 200-600 $\mu \mathrm{m} \times 500 \mu \mathrm{m}$ de $\varnothing$. Rostre composé de deux couronnes de 15 crochets de longueurs inégales (tableau VII).

TABLEAU VII. - Taille des crochets (en $\mu \mathrm{m}$ ) de C. laticollis chez les Avvicola d'Auvergne.

\begin{tabular}{ccccc}
\hline Crochets & Longueur & Hauteur & \multicolumn{2}{c}{$\begin{array}{c}\text { Longueurs* } \\
\text { du manche de la lame }\end{array}$} \\
& & & 186 & 172 \\
Grands & 328 à 385 & 98 & 135 \\
Petits & 230 à 260 & 80 & 109 & 135 \\
\hline
\end{tabular}

*mesurés à partir de l'extrémité inférieure de la garde.

Aspect histologioue. - Selon les exemplaires, la vésicule parasitaire est presque totalement libre dans la cavité kystique ou étroitement appliquée contre l'adventice. La structure de la paroi larvaire s'apparente à celle de $C$. tenvicollis. Le tégument limitant est fin $(1,5 \mu \mathrm{m})$; il acquiert des microtriches longs de 5 à $6 \mu \mathrm{m}$ lorsqu'il n'est pas en contact avec l'adventice. La couche parenchymateuse interne est épaisse au minimum de 20 à $30 \mu \mathrm{m}$; elle contient quelques solénocytes longs de $10 \times 4 \mu \mathrm{m}$, très inégalement répartis, à l'origine de canaux néphridiens dont les mieux figurés mesurent $10 \times 25 \mu \mathrm{m}$ de $\varnothing$ en section. A proximité de la zone d'implantation du scolex, tégument et parenchyme s'épaississent : le premier atteint $7 \mu \mathrm{m}$ et le second $200 \mu \mathrm{m}$. Ce dernier s'organise en travées rayonnantes serrées longues de $100 \mu \mathrm{m}$ et riches en noyaux. Une mince couche $(10 \mu \mathrm{m})$ de fibres musculaires apparaît de part et d'autre de l'implantation du scolex, à partir d'une distance de 1500 à $1000 \mu \mathrm{m}$; au-delà de cette couche, le parenchyme s'oriente de façon concentrique et constitue le matériel initial du pédicule du scolex.

Discussion. - La larve, de la taille d'un pois, vésiculeuse, liquidienne et monocéphalique, est caractéristique de la forme larvaire cysticerque. Celle-ci est inféodée aux cestodes de la famille des Taeniidae et plus précisément au genre Taenia Linné, 1758 et ses satellites. Dans le cas présent, s'en excluent les genres Multiceps, Hydatigera et Tetrathyrotaenia, car leurs larves se rattachent à un type morphologique différent (cénure, strobilocerque et tétrathyridium). Par ailleurs, l'existence d'un rostre invaginable armé de $2 \times 15$ crochets de grandes tailles et de longueurs inégales (>300 et $200 \mu \mathrm{m}$ ) empêche l'apparentement avec le genre Cladotaenia Cohn que de courts crochets $(<50 \mu \mathrm{m})$ caractérisent. Dans le genre Taenia, nombre et tailles des crochets de notre cysticerque ne restent compatibles, par le jeu des exclusions, qu'avec ceux de $T$. laticollis, parva et retracta; quant à macrocystis, la taille de ses petits crochets n'atteint qu'à peine la limite inférieure de la taille des crochets de l'espèce en discussion. Par ailleurs, des considérations de répartition géographique 
tendent à faire pratiquer l'élimination des espèces exotiques telles que macrocystis (Nouveau Monde), parva (Afrique) et retracta (Asie Centrale). T. laticollis s'impose dès lors comme la détermination la plus vraisemblable.

Le stade adulte du cestode existe en France chez la genette (Viverridé), le lynx ayant disparu. Son scolex est décrit comme volumineux (700 à $1900 \mu \mathrm{m}$ de $\varnothing)$, porteur d'un rostre invaginé déterminant une forte saillie antérieure, et constitué de deux couronnes de 19 à 21 crochets (jusqu'à 30) inégaux, mesurant 390-415 $\mu \mathrm{m}$ et 214-238 $\mu \mathrm{m}$ (in Abuladze, 1964). Les expériences de Joyeux et Baer, 1935 montrent que l'espèce évolue sous forme de cysticerque dans le foie de rongeurs. Toutes ces caractéristiques sont compatibles avec celles de la larve décrite; nous identifions donc cette dernière à Taenia laticollis Rud., 1819, et Arvicola terrestris constitue l'un de ses hôtes naturels en France. Ces derniers ne semblent pas avoir été décrits en Europe.

\section{4 - Hydatides alvéolaires de l'Echinococcus multilocularis}

L'identification des parasites du foie des Arvicola d'Auvergne constitue la corollaire de la recherche des hôtes intermédiaires de $E$. multilocularis dont l'importance médicale justifie une mention spéciale dans un travail séparé (cf. Pétavy et coll., $1983)$; il y est détaillé la description de ses multiples aspects. En résumé, la larve alvéolaire du tænia multiloculaire est observée 23 fois sur 943 autopsies (chez 10 mâles et 13 femelles adultes), soit chez 2,44\% des hôtes capturés, distribués dans 5 communes différentes (tableau VIII) ; la larve alvéolaire s'associe dans deux cas à un

TABleAu VIII. - Prévalence géographique des hydatides alvéolaires observées en 1981-1982 ches les Arvicola d'Auvergne.

\begin{tabular}{|c|c|c|c|c|c|c|c|}
\hline \multirow{2}{*}{$\begin{array}{l}\text { Dépar- } \\
\text { tements }\end{array}$} & \multirow{2}{*}{ Communes } & Dat & & Nombre & Foies & parasités & $\%$ \\
\hline & & \multicolumn{3}{|c|}{ des captures } & Nombre & $\%$ local & général \\
\hline \multirow{5}{*}{$\begin{array}{c}\text { Puy- } \\
\text { de- } \\
\text { Dôme }\end{array}$} & Voingt & Août & 1981 & 50 & 0 & - & \multirow{7}{*}{$2,44 \%$} \\
\hline & Giat & Mars & 1982 & 3 & 0 & - & \\
\hline & Mareuge & $\begin{array}{l}\text { Avril } \\
\text { Juill. }\end{array}$ & $\begin{array}{l}1982 \\
1982\end{array}$ & $\begin{array}{l}93 \\
94\end{array}$ & $\begin{array}{l}1 \\
0\end{array}$ & 0,53 & \\
\hline & Zanières & $\begin{array}{l}\text { Août } \\
\text { Mars }\end{array}$ & $\begin{array}{l}1981 \\
1982\end{array}$ & $\begin{array}{r}36 \\
161\end{array}$ & $\left.\begin{array}{l}0 \\
1\end{array}\right\}$ & 0,50 & \\
\hline & $\begin{array}{l}\text { Égliseneuve } \\
\text { d'Entraigues }\end{array}$ & Juill. & $\begin{array}{l}1982 \\
1982\end{array}$ & $\begin{array}{l}33 \\
57\end{array}$ & $1 !$ & 2,22 & \\
\hline \multirow[t]{2}{*}{ Cantal } & Allanche & $\begin{array}{l}\text { Août } \\
\text { Oct. } \\
\text { Janv. } \\
\text { Mai }\end{array}$ & $\begin{array}{l}1981 \\
1981 \\
1982 \\
1982\end{array}$ & $\begin{array}{r}37 \\
167 \\
74 \\
87\end{array}$ & $\left.\begin{array}{c}1^{*} \\
0 \\
13 \\
3\end{array}\right\}$ & 4,65 & \\
\hline & Condat & $\begin{array}{l}\text { Mars } \\
\text { Mai }\end{array}$ & $\begin{array}{l}1982 \\
1982\end{array}$ & $\begin{array}{l}27 \\
24\end{array}$ & $\begin{array}{l}1^{*} \\
1\end{array}$ & 3,92 & \\
\hline
\end{tabular}


kyste de $C$. fasciolaris et dans un cas avec trois ; il ne semble pas exister d'interactions réciproques évidentes.

Les parasites sont observés pratiquement en toutes saisons. Deux des hydatides sont fertiles soit $8,69 \%$ de leur total. Une des larves fertiles n'engendre qu'un très petit nombre de protoscolex dont certains sont dégénérés ; l'autre, d'un type inhabituel rappelant une hydatide plurivésiculaire de E. gramulosus, en produit des milliers, tous bien vivants. Des 21 larves acéphalocystes, 14 soit $60,8 \%$ sont des larves jeunes monovésiculaires, d'une taille approximative comprise entre 300 et $1500 \mu \mathrm{m}$ de $\varnothing$; 7 soit $30,4 \%$ sont des larves âgées plurivésiculaires d'une taille approximative comprise entre 1,5 et $8 \mathrm{~mm}$ de $\varnothing$. La progression des stolons ou rhizoïdes du parasite à partir de la lésion primaire est généralement tardive (chez 5 des 7 larves âgées devenues plurivésiculaires), sauf exception (chez 2 des 14 larves jeunes monovésiculaires).

DiAgnostic DifFÉRENTIEL. - Les lésions hépatiques attribuées à l'espèce multilocularis ont toutes été déterminées sur coupes histologiques. En absence de protoscolex, de stolons exogènes et des alvéoles caractéristiques de l'espèce - cas le plus fréquent dans la série des larves étudiées (14/23 larves, soit 60,8\%) —, le diagnostic d'espèce a été porté d'après l'aspect de la membrane cuticulaire présente au niveau de l'adventice ou formant des arabesques caractéristiques dans la cavité primaire de la larve. Chez les Arvicola, cette membrane cuticulaire des larves jeunes est primitivement unistratifiée, mince ( 1,5 à $3 \mu \mathrm{m})$, réfringente, hyaline et chromophile. Elle offre toujours, en un point ou un autre de la coupe observée des festons, des soulèvements, des réplications ou des délaminations où elle se stratifie en deux ou trois assises au moins qui l'épaississent jusqu'à $10 \mu \mathrm{m}$ ou davantage. Elle n'est jamais ciliée ni doublée d'une assise de fibrilles. La membrane cuticulaire est accompagnée d'une membrane proligère filamenteuse discrète relativement peu nucléée et disposée en réseau plat qui se développe plus abondamment au creux des festons de cuticule.

La vésicule primaire montre parfois en certains points bien localisés et restreints de sa périphérie des zônes où membrane cuticulaire, non encore formée, et membrane proligère font place à un plasmode, à noyaux nucléolés volumineux et abondants, en contact direct avec les tissus réactionnels de l'hôte.

L'histologie comparée de la cuticule des trois espèces de cysticerques parasites des foies des mêmes campagnols tend à montrer que - au moins dans les formes mûres de ces larves, seules accessibles à nos observations, - cette structure ne présente aucune des caractéristiques mentionnées pour l'hydatide; on peut supposer que les formes immatures des mêmes cystiques ne font pas exception; en absence de matériel favorable dans notre série, ce point particulier n'a toutefois pas été contrôlé.

\section{Relations hôte-parasites : la réaction fibro-conjonctive du foie parasité.}

La réaction fibro-conjonctive de l'hôte est stéréotypée quelle que soit l'espèce du parasite qui l'engendre ; la seule variable est représentée par le degré d'intensité qu'atteignent les réactions tissulaires et l'épaisseur de leurs diverses couches. Une 
réaction intense n'est pas nécessairement l'apanage des hydatides. L'anatomopathologie montre en lisière des kystes : 1) le parenchyme hépatique sain, dont la disposition naturelle des travées est contrariée par l'extension du parasite ; 2) l'adventice. Celle-ci est constituée en périphérie d'une réaction lympho-histiocytaire souvent intense, à la suite de laquelle s'affirme la réaction fibro-conjonctive, d'abord discrète et lâche, rayonnante ou arciforme, puis devenant dense et concentrique aux abords du parasite. Cette adventice est parfois faiblement vascularisée. Elle subit souvent localement des altérations de son ordonnance sous forme : a) d'un envahissement de cellules géantes multinucléées, seules ou accompagnées d'une couche de grandes cellules palissadiques radiées ; b) d'ilots de nécrose cellulaire avoisinant c) des zones d'inclusion de massifs granulaires denses chromophiles, préfigurant la dégénérescence calcique. Dans le cas particulier des hydatides 1) les massifs granulaires soulèvent fréquemment la membrane cuticulaire et se retrouvent inclus entre les festons de cuticule ; 2) les stolons du parasite, figurés sous forme de plasmodes à gros noyaux nucléolés, se situent dans des tunnels creusés dans un tissu réactionnel fibro-conjonctif lâche et non au sein des travées hépatiques; la membrane cuticulaire apparaît secondairement en bordure du plasmode, au contact du conjonctif, sous forme de lignes fines, courtes et interrompues.

\section{5 - Cysticercus longicollis de la cavité péritonéale}

Un Arvicola de Zanières présente à l'autopsie plusieurs dizaines de cysticerques libres dans la cavité péritonéale. L'incidence du parasitisme se situe à $0,1 \%$ des Arvicola capturés; elle est particulièrement faible (Pl.I, fig. 4).

Les vésicules mesurent 2 à $3 \mathrm{~mm}$ de longueur $\times 1$ à $1,2 \mathrm{~mm}$ de $\varnothing$; elles sont oblongues, plus cylindroïdes qu'ovoïdes; leur couleur est blanchâtre et translucide, leur paroi laissant apparaître la tache correspondant au scolex invaginé. De rares larves sont bicéphales et d'une taille supérieure à celle des larves monocéphales. Le scolex invaginé présente deux couronnes de 16 à 17 crochets inégaux mesurant 114$139 \times 40-50 \mu \mathrm{m}$ et $146-183 \times 50 \mu \mathrm{m}$, et d'une silhouette en tout point identique à celle des crochets du cestode adulte.

Le grand nombre de cysticerques chez l'hôte s'explique par un phénomène naturel de multiplication asexuée par bourgeonnement externe des larves en évolution; les cysticerques d'une même source y sont généralement enkystés dans une prolifération de tissu conjonctif sous-cutané ou musculaire ; on peut aussi, mais plus rarement, les découvrir libres et séparés dans les cavités pulmonaires et péritonéales, comme chez l'Arvicola autopsié.

La larve infeste les renards d'Auvergne (cf Pétavy et coll., 1980) sous le nom de Taenia crassiceps, avec un taux de 17,39\%. Le faible pourcentage des Arvicola parasités laisse supposer qu'une ou plusieurs autres espèces d'hôtes vertébrés, proies des renards, jouent le rôle de réservoir de virus principal du parasite dans la région ; beaucoup d'autres espèces de petits rongeurs et la taupe sont d'ailleurs connus comme parfaitement réceptifs. 


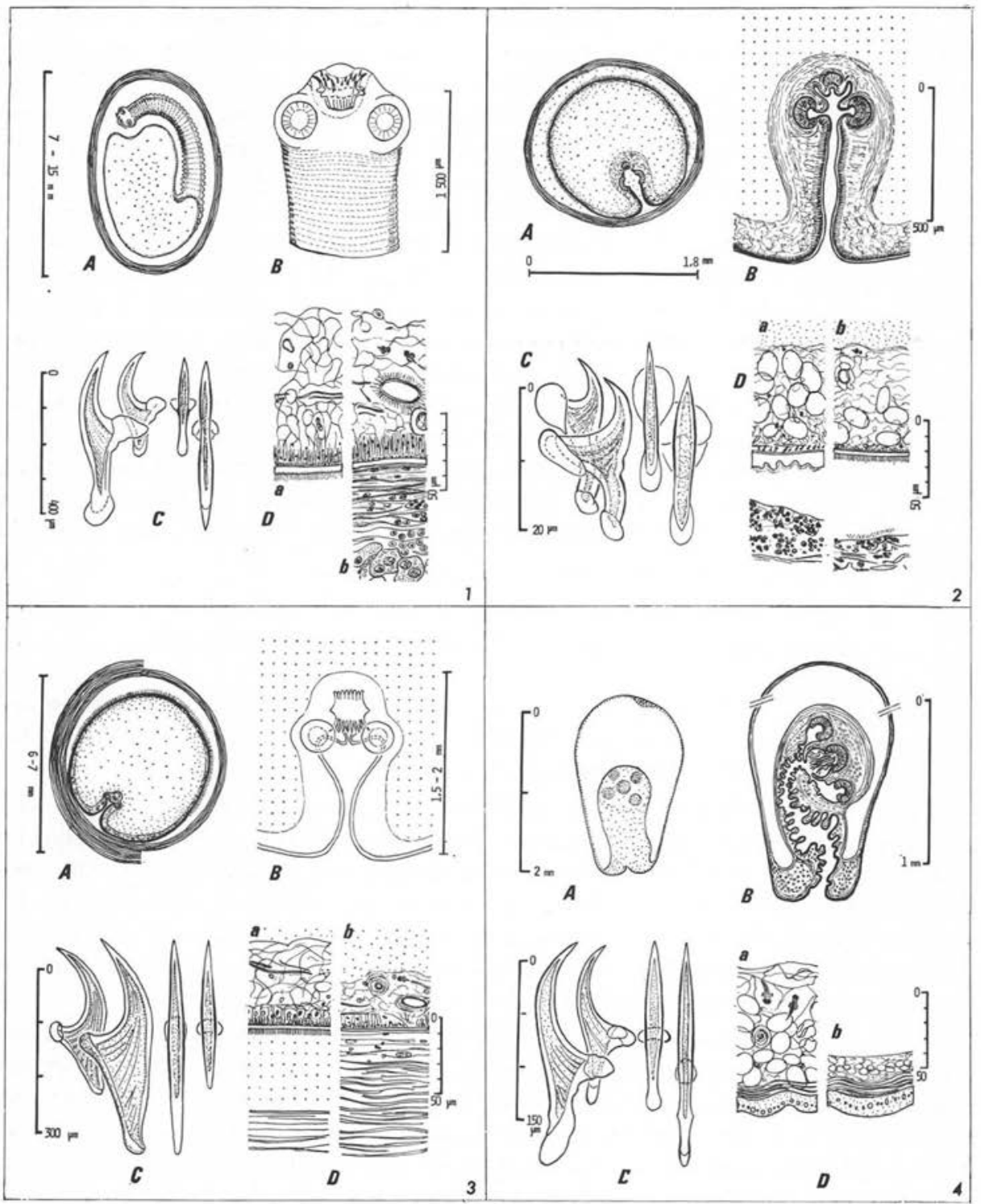

Planche I

FIG. I. - Cysticercus fasciolaris. A. Cystique schématique dans son kyste. - B. Scolex. - C. Crochets (profil et plan). - D. Paroi du cystique (coupe histologique) : (a) paroi libre ; (b) paroi en contact avec l'adventice.

FIG. 2. - Cysticercus tenuicollis : même légende qu'en I.

FIG. 3. - Cysticercus laticollis : même légende qu'en I.

FIG. 4. - Cysticercus longicollis : même légende qu'en I, sauf D. : paroi du cystique (a) à proximité du pôle d'invagination du scolex ; (b) au pôle opposé. 


\section{Clé dichotomique de détermination des larves de cestode parasites des Arvicola d'Auvergne}

\section{A - Parasites du foie}

1 - Cystiques bien circonscrits, ovoïdes ou subsphériques, formés d'une cavité liquidienne unique non cloisonnée intérieurement. Parois externes pourvues de

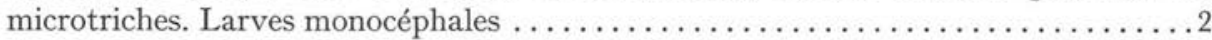

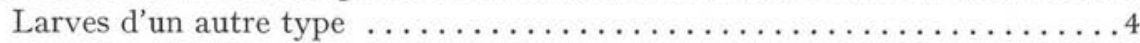

2 - Kyste généralement volumineux (entre 6 et $10 \times 15 \mathrm{~mm}$ de $\varnothing$ ) générateur d'une larve strobilisée de $2-3 \mathrm{~cm}$ jusqu'à $13-15 \mathrm{~cm}$, terminée par une vésicule. Scolex volumineux de $1200-1500 \mu \mathrm{m}$ de $\varnothing$ à deux couronnes de 15 à 18 crochets de très grande taille (430-470 et $250-290 \mu \mathrm{m})$..... Larve strobilocerque de Cysticercus fasciolaris.

Kyste sphérique d'une taille égale ou inférieure à 6-8 $\mathrm{mm}$ de $\varnothing$ générateur d'une larve monocéphale non strobilisée. Scolex interne à la vésicule porté par un cou court

3 - Kyste volumineux de 5 à $8 \mathrm{~mm}$ de $\varnothing$. Un scolex long de 800-1 $300 \times 600$ $700 \mu \mathrm{m}$; ventouses apparentes et petites $(160 \mu \mathrm{m}$ de $\varnothing)$; rostre invaginé sous un dôme saillant, armé de 2 couronnes de 15 grands crochets de tailles inégales (330-385 et

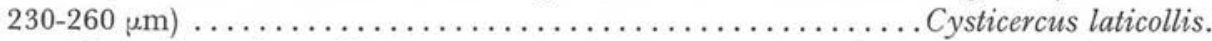

Kyste de petite taille $(1,8 \mathrm{~mm}$ de $\varnothing)$. Un scolex invaginé, en massue longue de $500 \times 200 \mu \mathrm{m}$ de $\varnothing$. Rostre armé de 2 petites couronnes de 23 crochets (18 à 32 in litt.) de petites tailles sub-égales $(19-23 \mu \mathrm{m})$. Ventouses inapparentes . . . . . . . . . . . . . ................... Cysticercus tenuicollis Rud., 1819 (= hypudaei Leuckart).

4 - Larves de tailles et d'aspects très variables selon le stade de maturation. Lésions hépatiques généralement très irrégulièrement et imparfaitement circonscrites. Un ou plusieurs lobes atteints. Larves fertiles ou non.

a) larves jeunes peu visibles dans le parenchyme ou sous-capsulaires, de petite taille (300 à $1000 \mu \mathrm{m}$ en général), uniloculaires avec tendance au cloisonnement interne de la cavité primaire par des festons de cuticule. Pas de scolex à ce stade.

b) larves âgées de $1 \mathrm{~mm}$ à $1 \mathrm{~cm}$ de $\varnothing$ ou davantage, formant des vésicules, des kystes ou des tâches jaunes polycycliques en surface du foie :

- les unes pluriloculaires. Production de stolons ou rhizoïdes plus ou moins tardive, dans un tissu fibreux réactionnel. Alvéoles vides ou pourvues de vésicules proligères engendrant des protoscolex. Essaimage à distance possible dans les autres lobes ;

- les autres pauciloculaires, à l'image des hydatides de l'espèce E. granulosus.

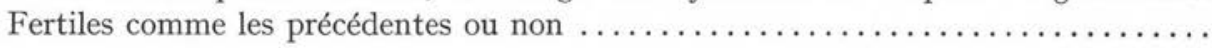




\section{B - Parasites du conjonetif ou des cavités}

Vésicules oblongues de 2 à $3,5 \mathrm{~mm} \times 1-1,2 \mathrm{~mm}$ de $\varnothing$ contenant un scolex invaginé et plus exceptionnellement deux. Rostre à deux couronnes de 16 à 17 crochets de tailles inégales (146-183 et $114-139 \mu \mathrm{m})$. Ventouses de $170 \times 135 \mu \mathrm{m}$ de $\varnothing$. Parasites enkystés en groupe dans le conjonctif ou libres dans la cavité pleurale ou péritonéale......... .Cysticercus longicollis.

\section{Résumé et conclusion}

Les foies de 943 Arvicola terrestris en Auvergne examinés en 1981 et 1982 présentent quatre espèces différentes de larves de cestodes, trois du type cysticerque et une du type hydatide. Leur fréquence figure dans le tableau $I X$, de même que celle d'un cystique de la cavité péritonéale.

Tableau IX. - Prévalence des larves de cestodes parasites du foie et de la cavité péritonéale de 943 Arvicola terrestris en Auvergne.

\begin{tabular}{lcc}
\hline \multicolumn{1}{c}{$\begin{array}{c}\text { Espèces de cestodes } \\
\text { hépatiques }\end{array}$} & $\begin{array}{c}\text { Nombre des } \\
\text { cas positifs }\end{array}$ & $\begin{array}{c}\text { Pourcentages des } \\
\text { hôtes parasités }\end{array}$ \\
\hline C. fasciolaris & 37 & 3,92 \\
C. tenuicollis & 11 & 1,16 \\
C. laticollis & 10 & 1,06 \\
E. multilocularis & 23 & 2,44 \\
\hline Cystique péritonéal : C. longicollis & 1 & 0,10 \\
\hline
\end{tabular}

Toutes les larves répertoriées sont celles de cestodes parasites de mammifères à l'exclusion de parasites d'oiseaux rapaces. Ces larves sont inégalement réparties dans le temps et l'espace des six communes prospectées (Voingt, Zanières, Mareuge, Égliseneuve d'Entraigues, Allanche et Condat).

C. fasciolaris est présent dans toutes les stations de piégeage et son endémie apparaît sensiblement stable dans le temps, au travers des saisons.

C. tenuicollis et $C$. laticollis ne sont présents que dans le centre de l'Auvergne, là où l'existence ou la densité de population des hôtes définitifs permet le maintien de l'endémie parasitaire.

C. multilocularis est distribué dans cinq des six communes prospectées avec des pourcentages locaux variant de $0,5 \%$ des prises à $4,65 \%$. Le parasite se maintient en toute saisons. Les hydatides jeunes et stériles prédominent (60\% des 23 cas) ; plusieurs larves âgées sont également stériles et deux seulement sont fertiles $(8,69 \%)$. 
L'une de celles-ci est du type alvéolaire classique envahissant plusieurs lobes mais dégénérative; elle est pauvre en protoscolex dont certains sont dégénérés ; l'autre est d'un type inhabituel, évoquant une larve plurivésiculaire de E. gramulosus, bien circonscrite dans un lobe et volumineuse; elle contient des milliers de protoscolex. Il apparaît que si $A$.terrestris est bien réceptif au parasite, il constitue, au moins en Auvergne, un hôte intermédiaire de médiocre qualité (maturation lente des larves et guérisons spontanées possibles).

\section{BIBLIOGRAPHIE}

AbUladze K. I. : Taeniata des animaux et de l'homme et affections provoquées. Éléments de Cestodologie, Skriabine ed., Izdatel. Nauka, 1964 (Moscou), IV, 530 p. (en russe et traduction anglaise).

Dollfys R. Ph. : Sur Taenia tenuicollis Rudolphi, I8I9 et son cystique. Ann. Parasitol. Hum. Comp., 1961, 36:384-396.

Joyeux C. Baer J. : Notices helminthologiques. Bull. Soc. Zool. France, 1935, 60, 482-501.

PÉtavy A. F., Deblock S. : Helminthes du renard commun (Vulpes vulpes L.) dans la région du Massif Central (France). Ann. Parasitol. Hum. Comp., 1980, 55, 379-391.

PÉtavy A. F., Deblock S. : Connaissance du foyer auvergnat d'échinococcose alvéolaire. Recherche de l'hôte intermédiaire. Description des lésions, Ann. Parasitol. Hum. Comp., 1983, s8, $423-437$. 